\title{
Ground state cooling, quantum state engineering and study of decoherence of ions in Paul traps
}

\author{
F. Schmidt-Kaler, Ch. Roos, H. C. Nägerl*, H. Rohde, S. Gulde, \\ A. Mundt, M. Lederbauer, G. Thalhammer, Th. Zeiger, P. Barton, \\ L. Hornekaer**, G. Reymond, D. Leibfried, J. Eschner, and R. Blatt \\ Institut für Experimentalphysik, Universität Innsbruck, \\ Technikerstraße 25, A-6020 Innsbruck, Austria, \\ *Norman Bridge Laboratory of Physics, Caltech, Pasadena, USA \\ **Aarhus University, Denmark
}

November 17, 2018

\begin{abstract}
We investigate single ions of ${ }^{40} \mathrm{Ca}^{+}$in Paul traps for quantum information processing. Superpositions of the $\mathrm{S}_{1 / 2}$ electronic ground state and the metastable $\mathrm{D}_{5 / 2}$ state are used to implement a qubit. Laser light on the $S_{1 / 2} \leftrightarrow D_{5 / 2}$ transition is used for the manipulation of the ion's quantum state. We apply sideband cooling to the ion and reach the ground state of vibration with up to $99.9 \%$ probability. Starting from this Fock state $|n=0\rangle$, we demonstrate coherent quantum state manipulation. A large number of Rabi oscillations and a ms-coherence time is observed. Motional heating is measured to be as low as one vibrational quantum in $190 \mathrm{~ms}$. We also report on ground state cooling of two ions.
\end{abstract}

\section{Quantum information processing with trapped ions}

Trapped and laser cooled ions in Paul traps are used for an implementation of quantum information processing. Internal electronic states of individual ions serve to hold the quantum information (qubits) and an excitation of common vibrational modes provides the coupling between qubits, which is necessary for quantum logic operations between qubits, more specifically, for the realization of gate operations between two ions. The Cirac-Zoller proposal [1] requires that initially the ions are cooled to the ground state of motion and that the whole system can be coherently manipulated and controlled. The time scale of decoherence and coupling to the environment is required to be much smaller than the time scale of coherent manipulation. 
a)

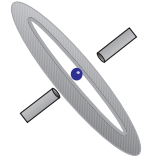

C)

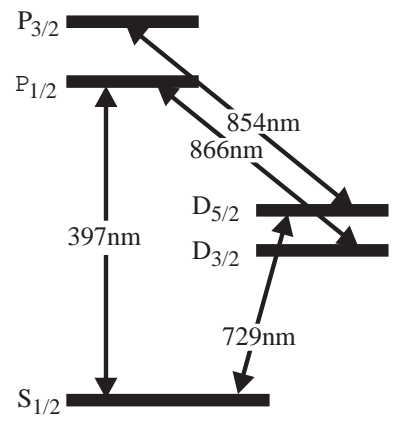

b)

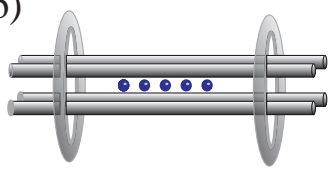

d)

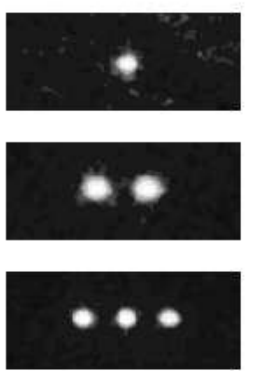

Figure 1: a) Spherical Paul trap, b) linear Paul trap, c) relevant energy levels of ${ }^{40} \mathrm{Ca}^{+}$ and the corresponding transition wavelengths, d) images of ion strings in the linear Paul trap observed by the intensified CCD camera. Here, the distance of two ions is $19 \mu \mathrm{m}$ at an axial trap frequency of $125 \mathrm{kHz}$.

The paper is organized as follows: In section II, we outline the techniques which are used for storing and detecting single ions in Paul traps. Section III decribes high resolution laser spectroscopy on the $\mathrm{S}_{1 / 2} \leftrightarrow \mathrm{D}_{5 / 2}$ transition, which is the basis of coherent manipulation of the internal electronic and external vibrational quantum state of a trapped ion. Ground state cooling for one and two ions is reported in section IV. For a single ion, we reached $99.9 \%$ of motional ground state occupation. Most recently, to scale up our results from one ion towards strings of ions, we have also shown ground state cooling for two ions. After a single ion is has been cooled, it is prepared for coherent manipulation (section $\mathrm{V}$ ) of the ion's vibrational state, including the generation of Fock states. We measured the coherence time of this process as well as the time scale of motional heating. Our experimental finding of $1 \mathrm{~ms}$ coherence time, and a heating rate of only one phonon in $190 \mathrm{~ms}$, shows good conditions for quantum information processing. Finally, in section VI, we investigate the maximum speed of gate operations and find that with our system approximately 30 - 50 gate operations are possible within the coherence time.

\section{Paul traps for single Calcium ions}

Ions are stored in Paul traps [2] under ultra high vacuum $\left(10^{-10 . .11} \mathrm{mbar}\right)$ conditions. In one of the experiments we use a spherical quadrupole Paul trap with a $1.4 \mathrm{~mm}$ ring diameter (Fig. 1.a) [3, where we observe motional frequencies $\left(\omega_{x}\right.$, $\left.\omega_{y}, \omega_{z}\right) /(2 \pi)$ of up to $(2.16,2.07,4.51) \mathrm{MHz}$ along the respective trap axes. The axial direction of the trap is denoted by $z$, the degeneracy of radial directions $x$ and $y$ is lifted by small asymmetries of the setup. Strings of ions are held in a linear trap (Fig. 1.b) [ [. 5], where we typically reach motional frequencies of $\left(\omega_{x}, \omega_{y}, \omega_{z}\right) /(2 \pi)=(2.0,2.0,0.7) \mathrm{MHz}$, e.g. three ions crystallize at an axial distance of $7 \mu \mathrm{m}$. 
${ }^{40} \mathrm{Ca}^{+}$ions have a single valence electron and no hyperfine structure. All relevant transitions are accessible by solid state or diode lasers (see Fig. 1.c) [3]. In our experiment, we apply Doppler cooling to the ion and detect the internal state on the $\mathrm{S}_{1 / 2}$ to $\mathrm{P}_{1 / 2}$ transition at $397 \mathrm{~nm}$, excited with a frequencydoubled Ti:Sapphire laser. This transition has a natural linewidth of $20 \mathrm{MHz}$ and is not closed since the ion may decay to the metastable $\mathrm{D}_{3 / 2}$ level. A diode laser at $866 \mathrm{~nm}$ serves to repump the ion via the $\mathrm{P}_{1 / 2}$ state thus closing the cooling cycle. As the upper internal level for quantum state engineering and sub-Doppler cooling, we employ the metastable $\mathrm{D}_{5 / 2}$ level with a natural lifetime of approx. $1 \mathrm{~s}$. The $\mathrm{S}_{1 / 2} \leftrightarrow \mathrm{D}_{5 / 2}$ quadrupole transition at $729 \mathrm{~nm}$ is excited with a Ti:Sapphire laser. We can detect whether a transition to $\mathrm{D}_{5 / 2}$ occurred by applying the beams at $397 \mathrm{~nm}$ and $866 \mathrm{~nm}$ and monitoring the fluorescence of the ion (electron shelving technique). The internal state of the ion is discriminated with an efficiency close to $100 \%$ [7]. Another diode laser at $854 \mathrm{~nm}$ is used to repump the ion from the $\mathrm{D}_{5 / 2}$ level to the electronic ground state via the $\mathrm{P}_{3 / 2}$ level. We observe the ions' fluorescence on a photomultiplier and an intensified CCD camera.

\section{Spectroscopy}

For coherent spectroscopic investigation and state engineering on the narrow $\mathrm{S}_{1 / 2} \leftrightarrow \mathrm{D}_{5 / 2}$ transition at $729 \mathrm{~nm}$ we use a pulsed technique which consists of five consecutive steps.

1. Laser light at $397 \mathrm{~nm}, 866 \mathrm{~nm}$, and $854 \mathrm{~nm}$ is used to pump the ion to the $\mathrm{S}_{1 / 2}$ ground state. At the Doppler limit, $E=\hbar \Gamma / 2[\mathbb{8}$, the thermal vibrational state corresponds to a mean vibrational quantum number $\langle n\rangle \cong 10$ for $\omega=(2 \pi) 1 \mathrm{MHz}$.

2. A constant magnetic field of a few Gauss splits the 10 Zeeman components of the $\mathrm{S}_{1 / 2} \leftrightarrow \mathrm{D}_{5 / 2}$ transition in frequency space. The $\mathrm{S}_{1 / 2}(m=-1 / 2)$ sub-state is prepared by optical pumping with $\sigma^{-}$radiation at $397 \mathrm{~nm}$.

3. Optional sideband cooling step (see also section 4$)$ : The $\mathrm{S}_{1 / 2}(m=$ $-1 / 2) \leftrightarrow \mathrm{D}_{5 / 2}(m=-5 / 2)$ transition is excited on one of the red sidebands. Therefore, the laser frequency is detuned red by one motional frequency $\left(\omega_{\text {laser }}=\omega_{S-D}-\omega_{\text {trap }}\right)$. The laser power is chosen so that approximately $1 \mathrm{~mW}$ laser power is focused to a waist size of $30 \mu \mathrm{m}$ (spherical trap), or $6 \mu \mathrm{m}$ (linear trap). The laser at $854 \mathrm{~nm}$ is switched on to broaden the $\mathrm{D}_{5 / 2}$ level at a power level which is set for optimum cooling. Optical pumping to the $\mathrm{S}_{1 / 2}(m=+1 / 2)$ level is prevented by interspersing short laser pulses of $\sigma^{-}$-polarized light at $397 \mathrm{~nm}$. The duration of those pulses is kept at a minimum to prevent unwanted heating.

4. Spectroscopy, or alternatively, state engineering step: We excite the $\mathrm{S}_{1 / 2}(m=-1 / 2) \leftrightarrow \mathrm{D}_{5 / 2}(m=-5 / 2)$ transition at $729 \mathrm{~nm}$ with a single laser pulse, or a number of pulses of well controlled frequency, power, 


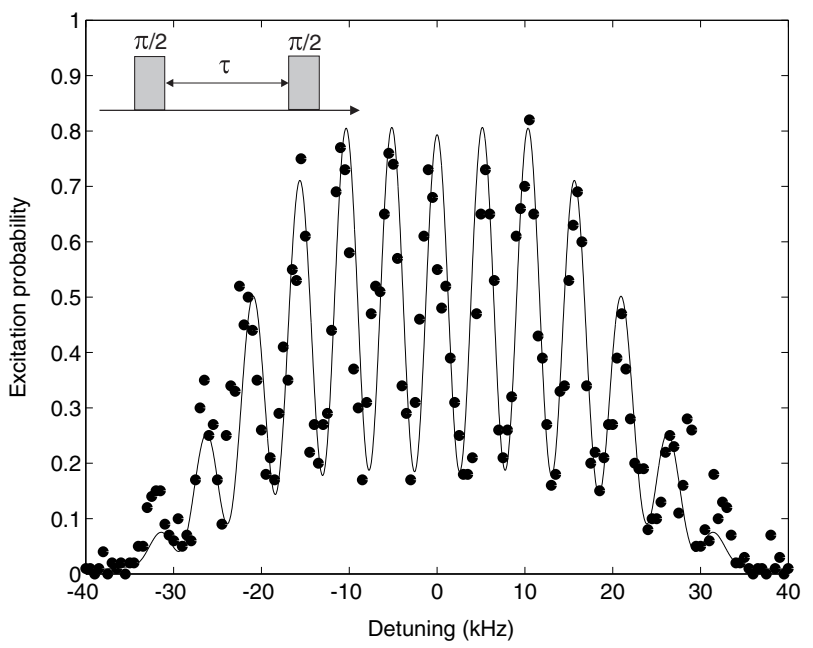

Figure 2: Ramsey spectroscopy of a single ion on the $\mathrm{S}_{1 / 2}(m=$ $-1 / 2) \leftrightarrow \mathrm{D}_{5 / 2}(m=$ $-5 / 2$ ) carrier transition. The time between pulses was $0.2 \mathrm{~ms}$ and each pulse length was $22 \mu \mathrm{s}$. From the fit, we deduce that the excitation pulse length was too long for a $\pi / 2$ pulse by $\sim 10 \%$ and the purely transversal decay constant is $\sim 2 \mathrm{kHz}$.

and timing. These parameters are chosen according to the desired state manipulation.

5. Final state analysis: The ion's fluorescence is collected under excitation with laser light at $397 \mathrm{~nm}$ and $866 \mathrm{~nm}$ and thus we detect whether a transition to the shelving level $\mathrm{D}_{5 / 2}$ has been previously induced.

This sequence is repeated typically 100 times to measure the $\mathrm{D}_{5 / 2}$ state population $P_{D}$ after the state engineering step. We study the dependence of $P_{D}$ on the experimental parameters, such as the detuning $\delta \omega$ of the light at $729 \mathrm{~nm}$ with respect to the ionic transition, or the length of one of the excitation pulses in step four. The duration of a single sequence is typically $20 \mathrm{~ms}$, so we can synchronize the sequence with the ac line frequency at $50 \mathrm{~Hz}$ to reduce acmagnetic field fluctuations.

A detailed study of the Zeeman and vibrational structure of the $\mathrm{S}_{1 / 2} \leftrightarrow$ $\mathrm{D}_{5 / 2}$ transition for a single ion in the linear trap was performed by Nägerl et. al. [5]. For the carrier transition (electronic excitation only, $n \rightarrow n$ ) we measure a $1 \mathrm{kHz}$ linewidth. We attribute this residual broadening to laser and magnetic field fluctuations.

Recently, we excited Ramsey fringes using two consecutive pulses. See Fig. 2 for the Ramsey signal of the $\mathrm{S}_{1 / 2} \leftrightarrow \mathrm{D}_{5 / 2}$ carrier transition for a single ion in the spherical Paul trap. Ramsey spectroscopy allows us to investigate the coherence of superposition states $\frac{1}{\sqrt{2}}\left\{\left|S_{1 / 2}\right\rangle+\left|D_{5 / 2}\right\rangle\right\}$ which are excited with the first pulse. The fit to the data of Fig. 2, allows us to estimate the purely transversal decay constant to be $2 \mathrm{kHz}$. We attribute this value to laser phase fluctuations and mechanical vibrations of the trap electrodes.

In the last part of this section we will focus on spectroscopy of ion crystals: Ions in a string are strongly coupled by the Coulomb interaction. Small displacements from their equilibrium positions are described in terms of normal modes 
of the entire ion crystal vibrating at distinct frequencies [6]. As an example, consider two ions confined in a Paul trap. The first normal mode corresponds to an oscillation of the entire crystal of ions moving back and forth as if they were rigidly joined. This oscillation is referred to as the center-of-mass mode (COM) [6]. The second normal mode, the so-called breathing mode, corresponds to an oscillation where the ions move in opposite directions. The frequency of this mode is at $\sqrt{3} \omega_{C O M}$.

In the experiment two ions were trapped in the spherical trap, and the excitation spectrum exhibits additional sideband resonances. Apart from the center-of-mass vibration frequencies, which remain at $\omega_{x}, \omega_{y}, \omega_{z}$, one observes additional sidebands which are identified in Fig. 3. The understanding of the full motional sideband structure presents the basis of further experiments, such as ground state cooling and driving coherent dynamics.

\section{Cooling to the vibrational ground state}

Preparation of the motional ground state is accomplished by a two-stage cooling process. First, the ion is pre-cooled on the $\mathrm{S}_{1 / 2}-\mathrm{P}_{1 / 2}$ dipole transition. In the second stage, a resolved-sideband cooling scheme, similar to the one used in Ref. [9], is applied on the narrow $\mathrm{S}_{1 / 2}-\mathrm{D}_{5 / 2}$ quadrupole transition: The laser frequency is detuned from the line center by the trap frequency, $\omega_{\text {laser }}=$ $\omega_{S D}-\omega_{\text {trap }}$ (first "red sideband" excitation), thus removing one phonon with each electronic excitation process. The cooling cycle is closed by a spontaneous decay to the ground state which conserves the phonon number in the LambDicke regime. When the vibrational ground state $|n=0\rangle$ is reached, the ion decouples from the laser excitation. Due to the weak coupling between light and atom on a quadrupole transition one would expect long cooling times. However, the cooling rate is greatly enhanced by (i) strongly saturating the transition and (ii) shortening the lifetime of the excited state via coupling to a dipole-allowed transition.

After sideband cooling, the ground state occupation is determined by probing the sideband absorption immediately after the cooling pulse. Fig. 4 shows the excitation probability $P_{D}$ for frequencies $\omega$ centered around the red and blue $\omega_{z}$ sidebands. For the quantitative determination of the vibrational ground state occupation probability $p(n=0)$ after sideband cooling, we compare $P_{D}$ for excitation at $\delta \omega=-\omega$ and $\delta \omega=+\omega$, i.e. on the red and on the blue sideband. For a thermal phonon probability distribution $p(n)$, the ratio of excitation on the red and blue sidebands is given by $P_{\text {red }} / P_{\text {blue }}=\langle n\rangle /(1+\langle n\rangle)$. A Lorentzian fit of the sideband heights yields a ground state occupation of $p_{0}=99.9 \%$ for the axial mode when $\omega_{z}=(2 \pi) 4.51 \mathrm{MHz}$ [3]. By cooling the radial mode with $\omega_{y}=(2 \pi) 2 \mathrm{MHz}$, we transfer $98 \%$ of the population to the motional ground state. Ground state cooling is also possible at lower trap frequencies, however slightly less efficient. At trap frequencies of $\omega_{z}=(2 \pi) 2 \mathrm{MHz}, \omega_{y}=$ $(2 \pi) 0.92 \mathrm{MHz}$ we achieve $\left\langle n_{z}\right\rangle=0.95$ and $\left\langle n_{y}\right\rangle=0.85$, respectively. The $\mathrm{x}$-direction is left uncooled because it is nearly perpendicular to the cooling 


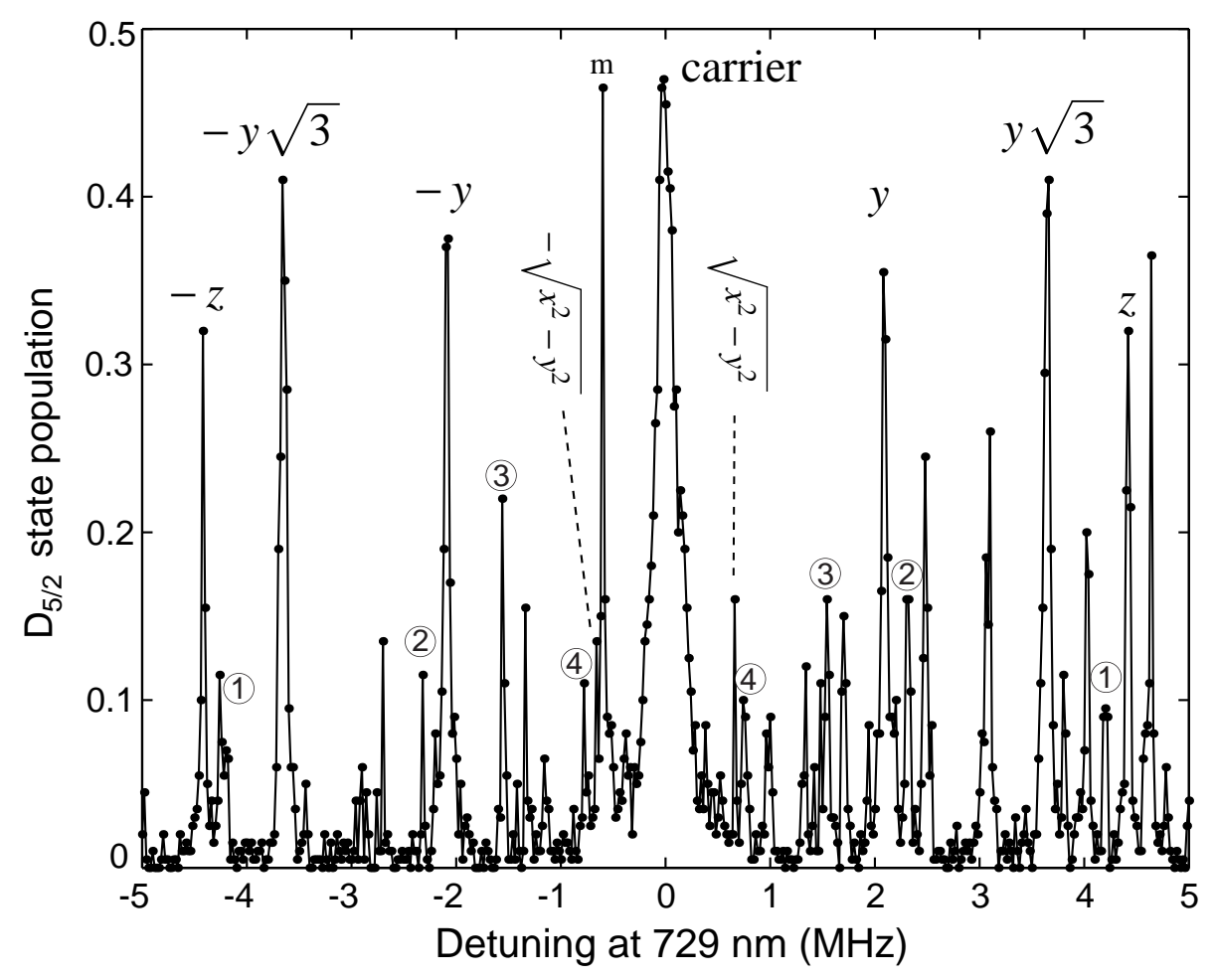

Figure 3: Sideband excitation spectrum on the $\mathrm{S}_{1 / 2}(m=-1 / 2) \leftrightarrow \mathrm{D}_{5 / 2}(m=$ $-5 / 2$ ) for two ions in the spherical trap. All motional sidebands are well understood in terms of normal modes: The $\omega_{y}, \omega_{z}, \sqrt{3} \omega_{y}$ and $\sqrt{\omega_{z}^{2}-\omega_{y}^{2}}$ sidebands are clearly visible. Numbered circles denote the second order sidebands, in detail (1): $2 \omega_{y},(2): \omega_{y}-\omega_{z},(3): \sqrt{3} \omega_{y}-\omega_{z},(4): \sqrt{3} \omega_{z}-\omega_{y}$. The x-direction is nearly at right angles to the $\mathrm{k}$ vector of the laser beam direction and thus only weakly excited. The line indicated with $(\mathrm{m})$ belongs to a different Zeeman transition. 

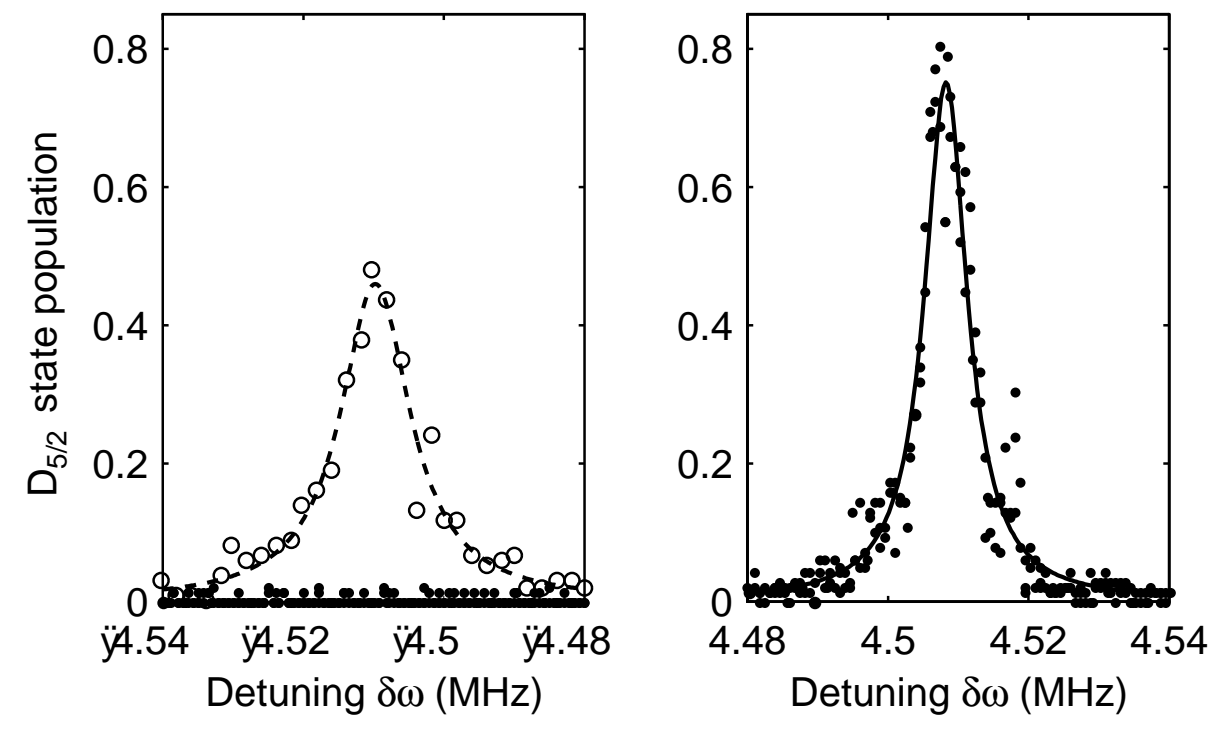

Figure 4: Sideband cooling of a single ion in the spherical Paul trap: Sideband absorption spectrum on the $\mathrm{S}_{1 / 2}(m=-1 / 2) \leftrightarrow \mathrm{D}_{5 / 2}(m=-5 / 2)$ transition after sideband cooling (full circles). The frequency is centered around the (left) red and (right) blue sidebands at $\omega_{z}=4.51 \mathrm{MHz}$. Open circles in the left hand diagram show the red sideband after Doppler cooling only. Each data point represents 400 individual measurements. The analyis yields a $99.9 \%$ ground state population. 
beam. We also succeeded in simultaneously cooling all three vibrational modes by using a second cooling beam and alternating the tuning of the cooling beams between the different red sidebands repeatedly [3]. For two ions in the spherical trap we achieved ground state cooling on the center-of-mass mode in the y and $\mathrm{z}$ directions, and on the breathing-mode with more than $96 \%$ ground state probability.

In the linear trap, our goal is to implement small scale quantum processing, thus, ions have to be kept apart so that they can be individually addressed. Thus, the laser beam at $729 \mathrm{~nm}$ is tightly focused into the linear Paul trap. We have demonstrated individual manipulation of single ions in strings [11. However, the addressing resolution is restricted by the quality of the optical system to $6 \mu \mathrm{m}$. If we demand individual addressing, this resolution restricts our ion - ion distances to greater than $6 \mu \mathrm{m}$, which corresponds to axial trap frequencies below $700 \mathrm{kHz}$ (with up to three ions). This low trap frequency aggravates the difficulty of ground state cooling, since the corresponding Doppler cooling limit is close to 20 phonons. Still, we succeed in cooling the axial mode at $700 \mathrm{kHz}$ and reach $p_{0}=90 \%$ ground state. We also achieved $95 \%$ in the ground state for cooling on the "rocking mode" at $\omega_{\text {rock }}=\sqrt{\omega_{\text {radial }}^{2}-\omega_{\text {axial }}^{2}}$, see Fig. 5. More advanced techniques, such as pre-cooling on the second sideband, prior to the usual first sideband cooling, will probably further increase the ground state occupation.

\section{Coherent manipulation}

Starting from the vibrational ground state, arbitrary quantum states can be created. For a demonstration of coherent state engineering, and in order to investigate decoherence, in step 4 we excite Rabi oscillations with the ion initially prepared in Fock states of its motion. Radiation at $729 \mathrm{~nm}$ is applied on the blue sideband transition $\left|\mathrm{S}, n_{z}\right\rangle \leftrightarrow\left|\mathrm{D}, n_{z}+1\right\rangle$ for a given interaction time $\tau$ and the excitation probability $P_{D}$ is measured as a function of $\tau$ [3]. The Rabi flopping behaviour allows us to analyse the purity of the initial state and its decoherence [12]. Fig. 5a shows $P_{D}(\tau)$ for the $|n=0\rangle$ state prepared by sideband cooling. Rabi oscillations at $\Omega_{01}=(2 \pi) 21 \mathrm{kHz}$ are observed with high contrast indicating that coherence is maintained for times above $1 \mathrm{~ms}$. For the preparation of the Fock state $|n=1\rangle$, we start from $|\mathrm{S}, n=0\rangle$, apply a $\pi$-pulse on the blue sideband and an optical pumping pulse at $854 \mathrm{~nm}$ to transfer the population from $|\mathrm{D}, n=1\rangle$ to $|\mathrm{S}, n=1\rangle$. As shown in Fig. $5 \mathrm{~b}$ for the $|n=1\rangle$ initial state, we also observe Rabi oscillations with a high contrast, now at $\Omega_{12}=(2 \pi) 30 \mathrm{kHz}$. The Rabi frequencies have been theoretically investigated and Blockley et. al. found $\Omega_{n, n+1} \propto \sqrt{n+1}$ in the Lamb-Dicke regime [13], which is fulfilled in good approximation for our trap. For the ratio of Rabi frequencies $\Omega_{12} / \Omega_{01}$ in the case of Fock states $|n=0\rangle$ and $|n=1\rangle$ we thus expect $\sqrt{2}$. The experimental finding agrees with $\sqrt{2}$ within $1 \%$. The Fourier transform of the flopping signals also yields directly the occupation probabilities for the contributing Fock states $|n=0,1,2,3 \ldots\rangle][12$ and allows us to calculate the purity of the prepared and 


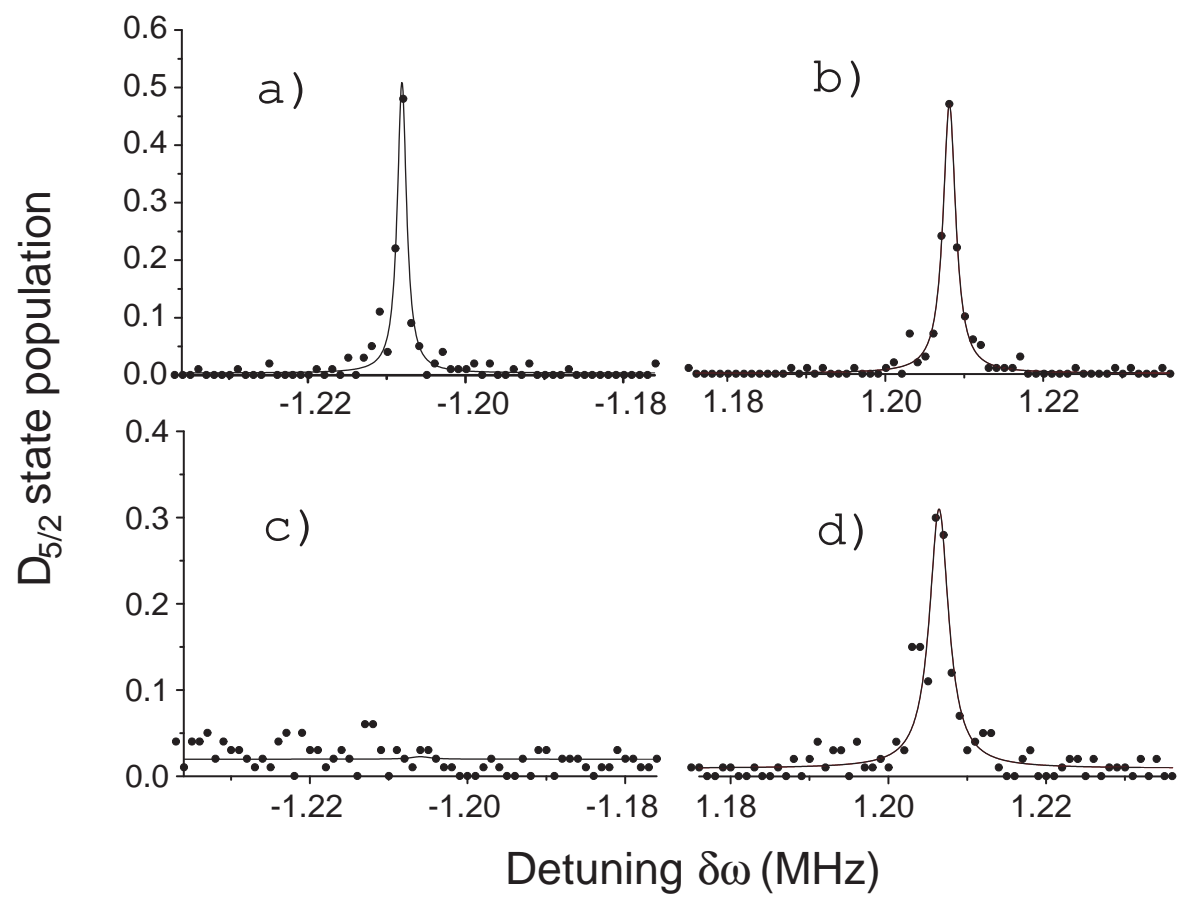

Figure 5: Sideband cooling of two ions in the linear Paul trap. Here, the ground state cooling of the "rocking" vibration mode is shown. The frequency of the rocking mode is calculated as $\omega_{\text {rock }}=\sqrt{\omega_{\text {radial }}^{2}-\omega_{\text {axial }}^{2}}=1.212 \mathrm{MHz}$, in good agreement with the experimental finding of $1.208 \mathrm{MHz}$. We plot the excitation probability to the $D_{5 / 2}$ state near the sideband versus the frequency detuning of the laser at $729 \mathrm{~nm}$. In the four plots, the frequency is centered around the red (a, c) and the blue (b, d) sideband of the rocking mode. Data (a, b) show the sideband excitation without, (c, d) with sideband cooling. Each data point represents 100 individual measurements. Lorentzians are fitted to the data and we obtain a ratio $P_{\text {red }} / P_{\text {blue }}$ which results in $p_{0}=95(4) \%$ ground state population. We achieve cooling on all radial and axial vibration modes for two ions, and measure heating rates of $\approx 20-50 \mathrm{~ms}$ per phonon. 


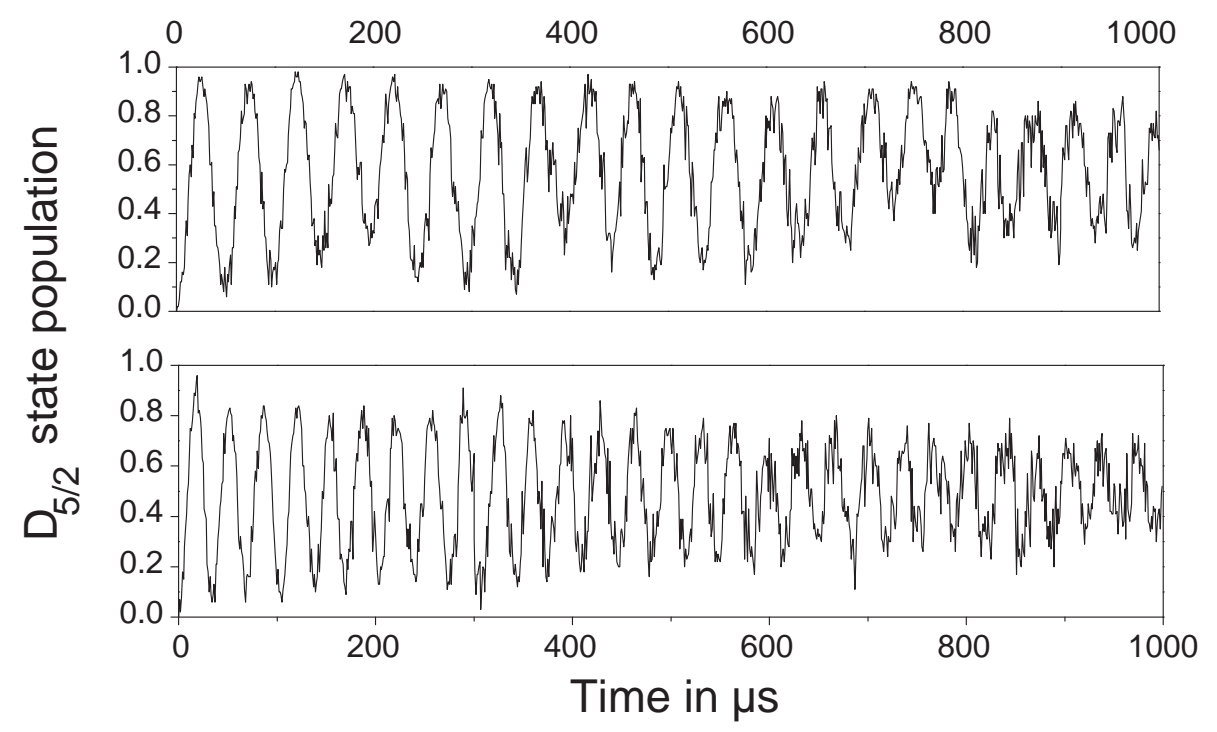

Figure 6: upper: Rabi oscillations of a single ion in the 3D trap on the blue sideband for the initial state $|n=0\rangle$. Coherence is maintained for up to $1 \mathrm{~ms}$. lower: Rabi oscillations as in the upper trace, but for an initial vibrational Fock state $|n=1\rangle$.

manipulated states. For the 'vacuum' state $|n=0\rangle$, we obtain $p_{0}=0.89(1)$ with impurites of $p_{1}=0.09(1)$ and $p_{n \geq 2} \leq 0.02(1)$. For the Fock state $|n=1\rangle$ the populations are $p_{0}=0.03(1), p_{1}=0.87(1), p_{2}=0.08(2)$, and $p_{n \geq 3} \leq 0.02(1)$. The measured transfer fidelity is about 0.9. Note that the Rabi flopping data here were taken with less efficient cooling (at a lower trap frequency), and the number state occupation from the Fourier analysis is consistent with the occupation which we determined by sideband measurements.

\section{$6 \quad$ Speed limits of gate operations}

As a figure of merit for an ion trap quantum processor, we calculate how many gate operations are feasible within the time scale given by decoherence. We have investigated the speed limit of quantum gate operations. The proposed methods [1, 15] employ the use of laser pulses to entangle the electronic and the vibrational degrees of freedom of trapped ions. A theoretical investigation shows that the proposed methods are limited mainly by the recoil frequency of the relevant electronic transition [14]. We have experimentally studied the basic building block of a gate operation, that is a $\pi$-pulse excitation on the first blue sideband. If the blue sideband is driven significantly faster than the inverse of the recoil frequency, carrier transitions are excited off-resonance and 
the contrast of the observed Rabi oscillations decreases. In the case of the quadrupole $\mathrm{S}_{1 / 2} \leftrightarrow \mathrm{D}_{5 / 2}$ transition we find a time of about $16 \mu \mathrm{s}$ for this limit, if we demand a gate fidelity of $99 \%$. For further details on fast gates, we refer to A. Steane et. al. [14]. We thus estimate that $30-50$ quantum gate operations will be feasible with the current setup.

\section{Conclusion}

We have engineered the quantum states of motion $|n=0,1\rangle$ of a single trapped ion that are relevant for quantum computation, using laser excitation on a forbidden optical transition. We have observed more than 30 periods of Rabi oscillations on the motional sidebands of this transition, thus showing that decoherence is negligible on the time scale of a few oscillations, i.e. the time required for a quantum gate operation. We attribute the observed $1 \mathrm{~ms}$ decoherence time to laser and magnetic field fluctuations. Heating of the motional degrees of freedom has also been observed and was measured directly to happen at least 1 order of magnitude more slowly. This confirms that in the comparatively large traps which we use, heating seems not to be a limiting process.

Furthermore, we have been able to cool two ions to the ground state, and also to reach the ground state for single and two ions in the linear trap, under conditions which allow individual addressing of ions in a string. Multiple coherent gate operations with trapped ions seem well within experimental reach.

This work is supported by the Austrian "Fonds zur Förderung der wissenschaftlichen Forschung" within the project SFB15, and in parts by the European Commission within the TMR networks "Quantum Information" (ERBFMRX-CT96-0087) and "Quantum Structures" (ERB-FMRX-CT96-0077), and the "Institut für Quanteninformation GmbH".

\section{References}

[1] J. I. Cirac and P. Zoller, Phys. Rev. Lett. 74, 4091 (1995).

[2] W. Paul, Rev. Mod. Phys. 62, 531 (1990).

[3] Ch. Roos, Th. Zeiger, H. Rohde, H.C. Nägerl, J. Eschner, D. Leibfried, F. Schmidt-Kaler, R. Blatt, Phys. Rev. Lett. 83, 4713

[4] H.C. Nägerl, W. Bechter, J. Eschner, F. Schmidt-Kaler, and R. Blatt, Appl. Phys. B 66, 603 (1998).

[5] H. C. Nägerl, Ch. Roos, D. Leibfried, H. Rohde, G. Thalhammer, J. Eschner, F. Schmidt-Kaler, and R. Blatt, Phys. Rev. A 61, 023405 (2000).

[6] D. F. V. James, Appl. Phys. B 66, 181 (1998).

[7] W. Nagourney, J. Sandberg, and H. Dehmelt, Phys. Rev. Lett. 56, 2797 (1986); Th. Sauter, W. Neuhauser, R. Blatt, and P. E. Toschek, Phys. Rev. 
Lett. 57, 1696 (1986); J. C. Bergquist, R. G. Hulet, W. M. Itano, D. J. Wineland, Phys. Rev. Lett. 57, 1699 (1986).

[8] D. Wineland and W. Itano, Phys. Rev. A 20, 1521 (1979).

[9] F. Diedrich, J. C. Bergquist, W. M. Itano, and D. J. Wineland, Phys. Rev. Lett. 62, 403 (1989).

[10] C. Monroe, D. M. Meekhof, B. E. King, W. M. Itano, and D. J. Wineland, Phys. Rev. Lett. 75, 4714 (1995); B. E. King, C. J. Myatt, Q. A. Turchette, D. Leibfried, W. M. Itano, C. Monroe, and D. J. Wineland, Phys. Rev. Lett. 81, 1525 (1998).

[11] H. C. Nägerl, D. Leibfried, H. Rohde, G. Thalhammer, J. Eschner, F. Schmidt-Kaler, and R. Blatt, Phys. Rev. A 60, 145 (1999)

[12] M. Brune, F. Schmidt-Kaler, A. Maali, J. Dreyer, E. Hagley, J. M. Raimond, and S. Haroche, Phys. Rev. Lett. 76, 1800 (1996); D. M. Meekhof, C. Monroe, B. E. King, W. M. Itano, and D. J. Wineland, Phys. Rev. Lett. 76, 1796 (1996).

[13] C. A. Blockley, D. F. Walls, and H. Risken, Europhys. Lett. 17, 509 (1992); J. I. Cirac, R. Blatt, S. Parkins, and P. Zoller, Phys. Rev. A 49, 1202 (1994).

[14] A. Steane, Ch. Roos, D.Stevens, A. Mundt, D. Leibfried, F. Schmidt-Kaler, and R. Blatt, quant-ph/0003087, submitted.

[15] C. Monroe, et. al., Phys. Rev. A 55, R2489 (1997). 